\title{
RADIAL DISTRIBUTION FUNCTION ANALYSIS OF CARBON NANOTUBES
}

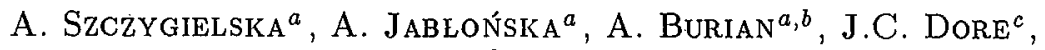 \\ V. HONKIMAKI ${ }^{d}$, AND J.B. NAGYe, \\ ${ }^{a}$ Institute of Physics, University of Silesia, Uniwersytecka 4, 40-007 Katowice, Poland \\ ${ }^{b}$ Centre of Polymer Chemistry, Polish Academy of Sciences \\ Marii Curie-Skłodowskiej 34, 41-819 Zabrze, Poland \\ ${ }^{c}$ School of Physical Sciences, University of Kent, Canterbury CT2 7NR, UK \\ ${ }^{d}$ European Synchrotron Radiation Facility, B.P. 220, 38043 Grenoble cedex 9, France \\ ${ }^{e}$ Institute for Studies in Interface Sciences, Facultes Universitaires Notre Dame de la Paix \\ rue de Bruxelles 61, 5000 Namur, Belgium
}

Wide-angle X-ray scattering studies of carbon nanotubes produced by catalytic decomposition of acetylene on a supported Co catalyst are reported. The X-ray intensities were recorded up to maximum scattering vector $K_{\max }=24 \AA$ and then Fourier transformed to real space. Yielding the radial distribution functions of good spatial resolution were analysed by the model based on fitting procedures method. Detailed analysis of the experimental data by a least-squares fitting procedure shows that the local atomic structure exhibits the expected hexagonal network with the nearest-neighbour $\mathrm{C}-\mathrm{C}$ distance of $1.41 \AA$. Investigation of stacking nature of subsequent layers suggests the structure in which adjacent layers are arranged without spatial correlations with inter-tubule spacing of about $3.4 \AA$.

PACS numbers: 61.10.-i, 81.05.Tp

\section{Introduction}

Carbon nanotubes were discovered in 1991 by Sumio Iijima and can be considered as cylindrical molecules consisting of graphene sheets wrapped up into the cylinders [1]. The diameter of carbon nanotubes ranges between 1 and $20 \mathrm{~nm}$ while typical lengths are about $1 \mu \mathrm{m}$. These materials have unique electrical and mechanical properties making them attractive for further applications. They have a light weight and extremely-high elastic modules, which are predicted to be by far the strongest carbon fibres that can be obtained. The conductive properties of nanotubes depend strongly on both the diameter and the chirality of the hexagonal carbon lattice. A slight change in the winding of hexagons along the tube can transform the tube from a metal into a semiconductor. 
The X-ray diffraction method was used to study spatial correlations between atoms in the investigated carbon nanotubes. In the present paper we address two questions: how atoms are arranged within a single tube and how the adjacent tubes are stacked? Two extreme models have been used for describing atomic arrangement - one with and second without graphitic inter-layer correlations.

\section{Experimental}

In the present work two main types of nanotubes were studied. The carbon nanotubes were prepared using a CVD method. They were produced by catalytic decomposition of acetylene on a supported Co catalyst. The samples were obtained by ultrasonication in toluene of the catalyst (nanotubes $A$ ) and by ultrasonication in toluene of the material which was obtained after oxidation of the $A$ type material in an aqueous solution of $\mathrm{KMnO}_{4} / \mathrm{H}_{2} \mathrm{SO}_{4}$ (nanotubes $B$ ). The preparation procedure was described in detail in Refs. [2] and [3].

The high-energy X-ray scattering experiments were performed on the ID15A beam-line at European Synchrotron Radiation Facility (ESRF, Grenoble). The photon energy $E=116.2 \mathrm{keV}(\lambda=0.1067 \AA)$ was used for the measurements. The samples were placed into capillary tubes of $3 \mathrm{~mm}$ diameter and mounted on the diffractometer axis. The intensities were recorded using a Ge solid-state detector. The measured intensities cover a $K$-range up to $24 \AA^{-1}$.

\section{Theoretical background}

For carbon nanotubes, the diffraction pattern arises from the coherent interferences of scattered waves from the distribution of identical scattering centres. Scattering by non-periodic systems are usually described in terms of the structure factor $S(Q)$ which is related to the radial distribution function (RDF) as follows:

$$
4 \pi r^{2} \rho_{0}\left[\rho(r)-\rho_{0}\right]=\frac{2 r}{\pi} \int_{0}^{K_{\max }} K[S(K)-1] \frac{\sin \left(\pi K / K_{\max }\right)}{\pi K / K_{\max }} \sin (K r) \mathrm{d} K,
$$

where $\rho_{0}$ is the average number density. The structure factor is defined as $I(K) / f^{2}$, where $I(K)$ is the corrected and normalised intensity and $f$ is the atomic scattering factor. The RDF provides information about the probability of finding an atom in a spherical shell at a distance $r$ from an arbitrary atom. Successive peaks correspond to nearest, second and next-neighbour atomic distribution. Now, the radial distribution function (RDF) can be expressed as

$$
R D F=4 \pi r^{2} \rho(r) \text {. }
$$

Assuming that there are no spatial correlations between successive tubes we can express the total RDF as the sum of two contributions arising from correlations within a single layer and from averaged inter-tubule correlations (the so-called turbostratic term). Thus the theoretical RDF can be written as 


$$
\begin{aligned}
& 4 \pi r^{2} \rho(r)=\underbrace{\ldots \ldots \ldots \ldots \ldots}_{\text {as for one layer }} \\
&+4 \pi r n_{a} \underbrace{\left\{\frac{\left[1-\exp \left(-d_{c} / L_{c}\right)\right]^{\left[r / d_{c}\right]}}{\exp \left(d_{c} / L_{c}\right)-1}-\frac{L_{c}}{d_{c}}\left[1-\exp \left(r / L_{c}\right)\right]\right\}}_{\text {turbostratic term }},
\end{aligned}
$$

where $n_{a}$ is the number of atoms per unit area on plane $\rho_{0}=n_{a} / d_{c}, d_{c}$ - the inter-tube spacing, $L_{c}$ is the mean crystallite height and $\left[r / d_{c}\right]$ denotes the greatest integer in $r / d_{c}$ [4].

\section{Results and discussion}

The X-ray intensity data for investigated samples were corrected using standard procedures. The structure factors $S(K)$ in the $K$-range from about $0.5 \AA^{-1}$ to $24 \AA^{-1}$ were then calculated. Beyond this $K$-range, the value of the atomic

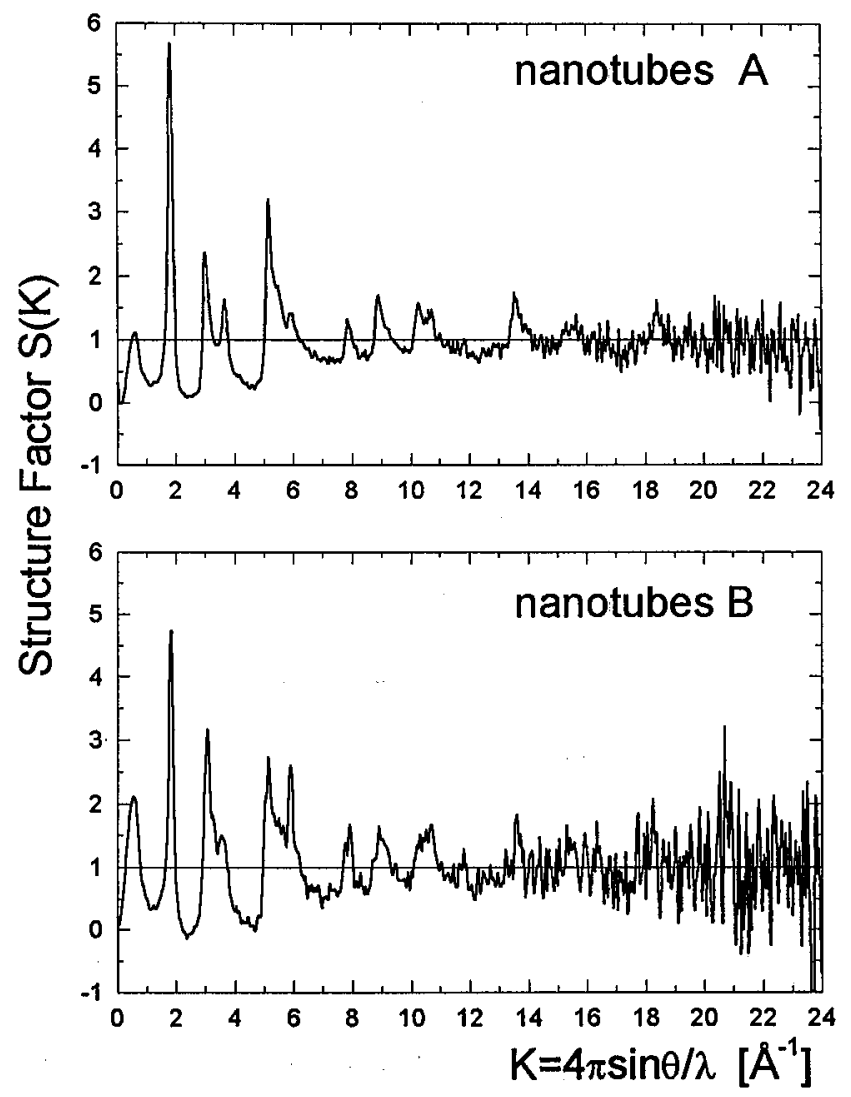

Fig. 1. The structure factors $S(K)$ obtained for investigated samples. 


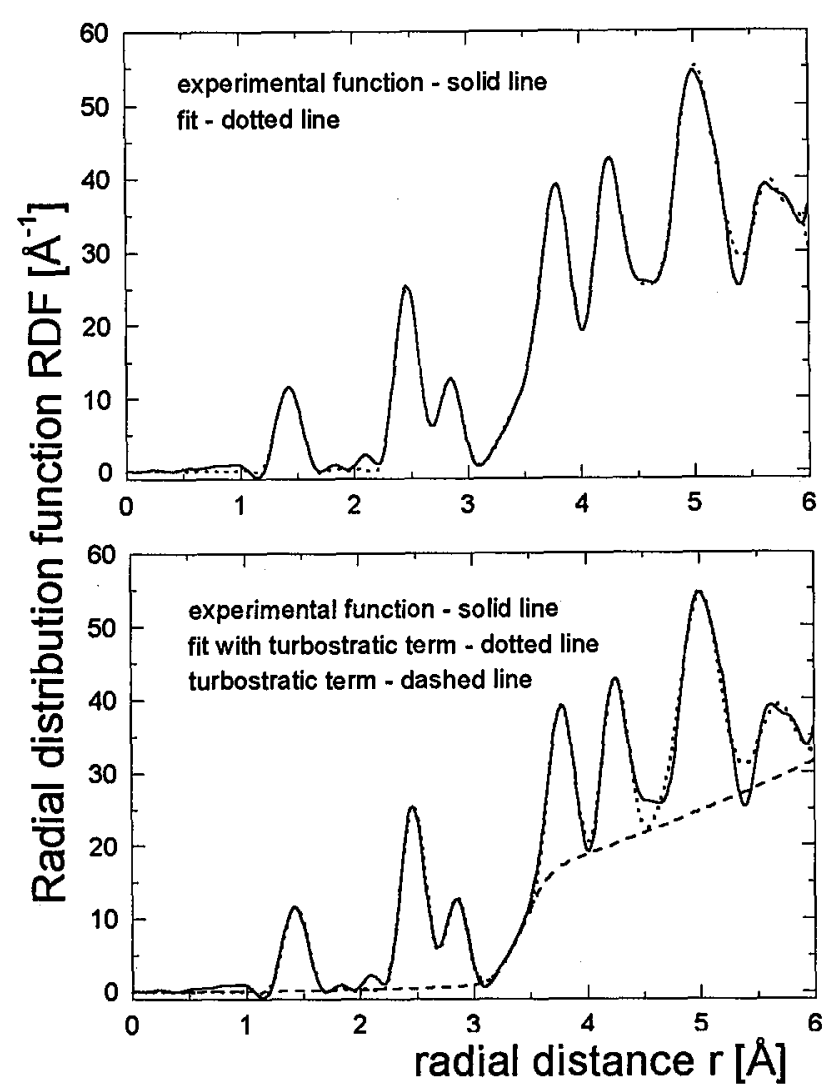

Fig. 2. Comparison of the calculated and experimental RDF's for the model without (top) and with turbostratic term (bottom) - nanotubes $A$.

scattering factor approaches zero and the measured intensities do not exhibit structure-related oscillations, instead, only noisy features are observed. The results are shown in Fig. 1. The RDF's were computed according to Eq. (1) in the $\mathrm{K}$-range from 0 to $24 \AA^{-1}$ for both investigated samples $A$ and $B$. Attempts have been made to simulate the experimental RDF's using a model without the baseline turbostratic contribution, taking the value of $\rho_{0}=0.114$ and the inter-tubule spacing of $d_{c}=3.43 \AA$. The interatomic distances $r_{i j}$, their standard deviation and the coordination numbers $N_{i j}$ were obtained using the curve-fitting method in the $r$-range of $0-6 \AA$. The calculated and experimental RDF's for nanotubes $A$ are compared in Fig. 2 (on the top).

This comparison shows that both amplitudes and peak positions can be reproduced by this model. The structural parameters $r_{i j}, N_{i j}$, and $\sigma_{i j}$ are listed in Table. However, the comparison of the parameters obtained for the investigated samples and those of graphite show generally that for the model without the turbostratic term, the coordination numbers $N_{i j}$ are higher than those characteristic of graphite. The nearest-neighbour interatomic distance corresponding to the $\mathrm{C}-\mathrm{C}$ 
TABLE

RDF peak positions $r$, the coordination numbers $N$ and standard deviation $\sigma$ of the interatomic distances for graphite and investigated nanotubes.

\begin{tabular}{|c|c|c|c|c|c|c|c|}
\hline \multirow{2}{*}{\multicolumn{2}{|c|}{ Graphite }} & \multicolumn{6}{|c|}{ Nanotubes A } \\
\hline & & \multicolumn{3}{|c|}{ Without turbostratic term } & \multicolumn{3}{|c|}{ With turbostratic term } \\
\hline$r[\AA]$ & $N$ & $r[\AA]$ & $N$ & $\sigma$ & $r[\AA]$ & $N$ & $\sigma$ \\
\hline 1.4180 & 3 & 1.4067 & 2.8765 & 0.0518 & 1.4067 & 2.8765 & 0.0518 \\
\hline 2.4560 & 6 & 2.4507 & 6.2831 & 0.0543 & 2.4507 & 6.2831 & 0.0543 \\
\hline 2.8359 & 3 & 2.8242 & 3.1033 & 0.541 & 2.8229 & 2.7672 & 0.0342 \\
\hline $3.3480^{a}$ & 2 & 3.4308 & 2.9214 & 0.1257 & - & - & - \\
\hline $3.6359^{a}$ & 6 & \multirow[b]{2}{*}{3.7696} & \multirow[b]{2}{*}{13.3736} & \multirow[b]{2}{*}{0.1158} & - & - & - \\
\hline 3.7516 & 6 & & & & 3.7548 & 5.6240 & 0.0586 \\
\hline $4.1522^{a}$ & 12 & \multirow[b]{2}{*}{4.2140} & \multirow[b]{2}{*}{12.0210} & \multirow[b]{2}{*}{0.0931} & - & - & - \\
\hline 4.2539 & 6 & & & & 4.2379 & 5.7846 & 0.0591 \\
\hline $4.3877^{a}$ & 6 & 4.5099 & 7.9629 & 0.1264 & - & - & - \\
\hline 4.9120 & 6 & \multirow[t]{3}{*}{4.9499} & \multirow[t]{3}{*}{20.4531} & \multirow[t]{3}{*}{0.1612} & 4.9786 & 12.4099 & 0.1481 \\
\hline $5.0283^{a}$ & 12 & & & & - & - & - \\
\hline 5.1126 & 6 & & & & & & \\
\hline $5.4134^{a}$ & 12 & 5.3072 & 14.7426 & 0.2266 & - & - & - \\
\hline 5.6719 & 3 & 5.6400 & 7.8864 & 0.0895 & 5.6541 & 3.7536 & 0.1296 \\
\hline $5.9445^{a}$ & 12 & 5.9242 & 10.8664 & 0.1107 & - & - & - \\
\hline
\end{tabular}

bond length is slightly shorter in nanotubes $A$. Similar fitting procedures were applied for the model with averaged inter-layer correlations between atoms according to Eq. (3). The results of such modelling, together with the turbostratic term, are shown in Fig. 2 (bottom). This model accounts very well for the experimental data and the coordination numbers within a single layer are close to the values typical of graphite. This analysis suggests that the local atomic structure can be described by the hexagonal network with the nearest-neighbour $\mathrm{C}-\mathrm{C}$ distance of $1.41 \AA$. The contribution to the RDF in the range $3.2-3.5 \AA$ is obvious, which indicates that the inter-layer correlations are, at least, locally preserved. However, the coordination numbers obtained for the turbostratic model are in better agreement with the values typical of intra-layer correlations than those of the model without the base line in comparison with graphite coordination. The analysis of these parameters proves that structure of nanotubes $A$ prefers a model with the turbostratic contribution.

In the case of nanotubes $B$, the structure factor $S(K)$ exhibits a strong peak at about $6 \AA^{-1}$. Moreover, the peak about $3 \AA^{-1}$ has apparently higher amplitude than that of nanotubes $A$. These peaks can be related to the presence 

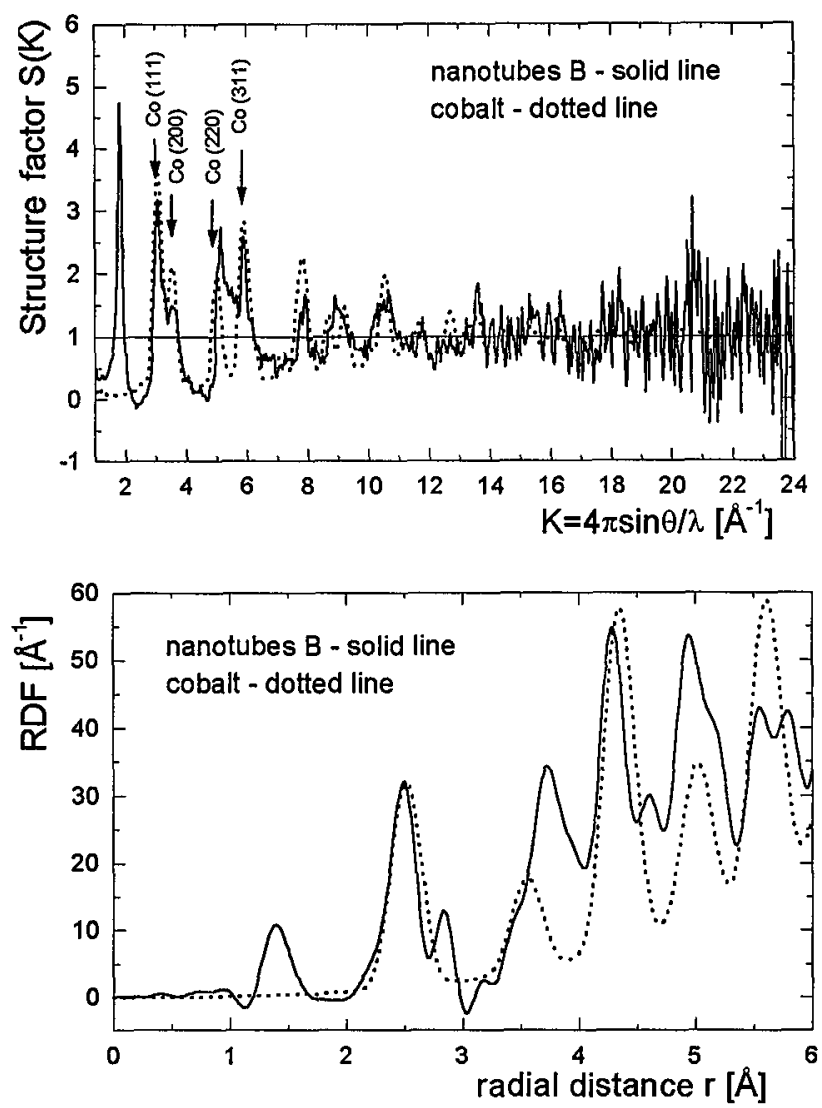

Fig. 3. Comparison of the structure factors $S(K)$ and RDF's for cobalt microcrystallites of $20 \AA$ in diameter and nanotubes $B$. The Miller indices of Co are also included.

of microcrystalline Co. That can be easily seen in Fig. 3 where computed $S(K)$ and $\mathrm{RDF}$ are compared with the relevant experimental functions. From this comparison one can conclude that the sample $B$ contains more Co microcrystallites.

\section{Conclusions}

In the present work two models of the structure of nanotubes have been tested. The obtained results demonstrate that the structure of nanotubes $A$ cannot be described in terms of the simple microcrystalline model, based on the graphite structure. The graphitic spatial correlations between adjacent layers may be retained only over a limited region. The obtained results show that the model in which spatial correlations between layers are averaged describes satisfactorily the $S(K)$ and RDF functions. Analysis of the experimental data in real and reciprocal spaces is a good test of a purity of the samples. 


\section{References}

[1] S. Iijima, Nature 354, 56 (1991).

[2] A. Fonseca, K. Hernadi, J.B. Nagy, D. Bernaerts, A. Lucas, J. Mol. Catal. 107, 159 (1995).

[3] T. Kyotani, Li-fu Tsai, A. Tomita, Chem. Mater. 8, 2109 (1996).

[4] A. Burian, A. Ratuszna, J.C. Dore, S.W. Howells, Carbon 36, 1613 (1998). 